Scientific Investigation in Dentistry - SID

ISSN 2447-8520

http://periodicos.unievangelica.edu.br/index.php/scientificinvestigationindestist

\title{
Relato de caso
}

\section{CONTRIBUIÇÃO DA TOMOGRAFIA COMPUTADORIZADA DE FEIXE CÔNICO NO DIAGNÓSTICO DE FRATURA RADICULAR: RELATO DE CASO}

\section{Contribution of cone-beam computed tomography in the diagnosis of root fracture: case report.}

\author{
Juliano Martins BUENO1, Fernando Fortes PICOLI ${ }^{2}$, Daniel de Almeida \\ DECÚRCIO ${ }^{3}$, Giulliano Caixeta SERPA ${ }^{4}$, Carolina Cintra GOMES ${ }^{5}$, Mayara \\ Barbosa Viandelli MUNDIM-PICOLI ${ }^{5}$.
}

\begin{abstract}
1. Radiologista e Diretor Científico do Centro Integrado de Radiodontologia - C.I.R.O. - Goiânia, Goiás, Brasil.
2. Mestre, Professor do Curso de Odontologia da Faculdade Evangélica de Goianésia - FACEG - Goianésia, Goiás, Brasil.

3. Doutor, Professor da Faculdade de Odontologia da Universidade Federal de Goiás - UFG - Goiânia, Goiás, Brasil.

4. Mestre, Professor do Curso de Odontologia do Centro Universitário de Anápolis - UniEVANGÉLICA - Anápolis, Goiás, Brasil.

5. Doutora, Professora do Curso de Odontologia do Centro Universitário de Anápolis - UniEVANGÉLICA - Anápolis, Goiás, Brasil.
\end{abstract}

\begin{tabular}{ll}
\hline Informação sobre o manuscrito & Autor para contato: \\
Recebido em: 01 Out 2018 & Prof $^{\text {a }}$ Mayara Barbosa Viandelli Mundim-Picoli \\
Aceito em: 28 Fev 2019 & Avenida Universitária, Qd.60, Lt.08 Edifício \\
& Torre Macedônia I, Vila Santa Isabel, Anápolis/ \\
& CEP 75083-350. \\
& E-mail: mayara.viandelli@gmail.com.
\end{tabular}

\begin{abstract}
RESUMO
Objetivo: As fraturas radiculares podem comprometer o dente, e se não forem corretamente diagnosticadas e tratadas, podem levar à exodontia do mesmo. Uma opção de diagnóstico é a tomografia computadorizada de feixe cônico (TCFC), permitindo a detecção, localização e visualização da extensão das fraturas. Este trabalho tem como objetivo apresentar um caso de aplicação da TCFC no diagnóstico de fratura radicular. Relato de Caso: Paciente 50 anos, compareceu ao dentista com queixa de dor provocada ao mastigar. A dor era localizada no dente 36 , e foi percebida há 3 meses, com discreto agravamento durante o período relatado. Foi relatado tratamento endodôntico no dente 36 há 1 ano. O exame intraoral revelou dor a percussão. Foi solicitada radiografia periapical que revelou presença de rarefação óssea periapical, em periodonto lateral e espessamento do ligamento periodontal no 36, além de perda vertical da crista óssea alveolar na sua face distal. Optou-se pela realização de uma TCFC do 36 para avaliar a possibilidade de fratura radicular e esta revelou imagem hiperdensa no interior dos condutos radiculares mésio-vestibular, mésio-lingual e distal compatível com material obturador e linha hipodensa longitudinal, no terço cervical até o apical, na raiz distal, compatível com fratura e perda óssea associada, com lesão em furca. Diante do quadro, optou-se pela exodontia do dente e reabilitação com implante. Uma radiografia periapical foi realizada 4 meses após a exodontia e notou-se sinais compatíveis com reparo ósseo. Considerações: $A$ TCFC contribuiu para o diagnóstico da fratura radicular, e estabelecimento do correto plano de tratamento para o caso.
\end{abstract}

PALAVRAS-CHAVE

Endodontia; Diagnóstico; Tomografia computadorizada por Raios $X$. 


\section{INTRODUÇÃO}

A fratura radicular, de maneira geral, se estende do canal radicular para região de periodonto, e pode ser iniciada tanto na coroa quanto na porção radicular, ou em alguns casos, no espaço entre coroa e raiz. Em se considerando a etiologia, a fratura radicular pode ser causada por diversos fatores, como instalação de retentores intrarradiculares, obturação radicular com pressão excessiva e restaurações com contatos prematuros ${ }^{1}$.

Um dos maiores desafios na rotina clínica para o cirurgião-dentista é o diagnóstico correto das fraturas radiculares. Caso ocorra falha em seu diagnóstico, o paciente pode ter sintomatologia dolorosa, estresse e muitas vezes, perda do elemento dental $^{2}$. Na maioria dos casos, o diagnóstico só é possível após um conjunto de sinais e sintomas clínicos, aliados às radiografias convencionais ou tomografia computadorizada ${ }^{3,4}$, ou através de cirurgias de inspeção'.

A radiografia periapical, por muito tempo, foi considerada o método de diagnóstico por imagem mais utilizado para a detecção de fraturas radiculares, e no momento atual, ainda é considerado um método adequado para visualização de coroa dentária, raiz e estruturas circundantes de suporte ${ }^{2}$. No entanto, a radiografia periapical apresenta limitações para o diagnóstico de fraturas radiculares, por apresentar visualização de uma imagem em duas dimensões onde a presença de estruturas anatômicas pode se sobrepor a linha de fratura ${ }^{2,3,5}$.

As fraturas radiculares verticais só são identificadas na radiografia periapical quando o feixe de raios-x se encontra paralelo à linha de fratura. Outra limitação da radiografia periapical está nos casos onde a fratura se encontra nos seus estágios iniciais, em que não houve separação dos fragmentos, tornando sua visualização dificultada 5 .

A tomografia computadorizada de feixe cônico (TCFC) foi introduzida na endodontia para a avaliação das características dentárias, como tamanho real dos condutos radiculares, extensão e destruição do osso alveolar e patologias periapicais. Também apresenta aplicabilidade para a avaliação da anatomia interna do sistema de canais radiculares, reabsorções e fraturas radiculares ${ }^{6}$.

A detecção de fraturas radiculares com o uso da TCFC tem sido considerada mais eficiente que a radiografia periapical convencional, pela possibilidade da navegação em uma imagem em três dimensões, em diferentes planos do espaço, melhorando a acurácia deste exame em comparação a radiografia convencional ${ }^{2,3,7}$.

Este trabalho tem como objetivo apresentar um relato de caso de aplicação da TCFC no diagnóstico de fratura radicular.

\section{RELATO DE CASO}

Uma paciente de 50 anos de idade, do gênero feminino, compareceu ao consultório odontológico com queixa de sintomatologia dolorosa provocada ao mastigar. A dor era localizada no dente 36 , e havia sido percebida há 3 meses, com discreto agravamento durante $\mathrm{o}$ período relatado. Era relatado histórico de tratamento endodôntico no dente 36 há 1 ano. 
O exame intra-oral revelou dor a percussão. Após exame clínico, foi solicitada radiografia periapical que revelou presença de rarefação óssea periapical, em periodonto lateral no dente 36 associada a espessamento do ligamento periodontal no terço cervical do mesmo dente, além de perda vertical da crista óssea alveolar na sua face distal (Figura 1).

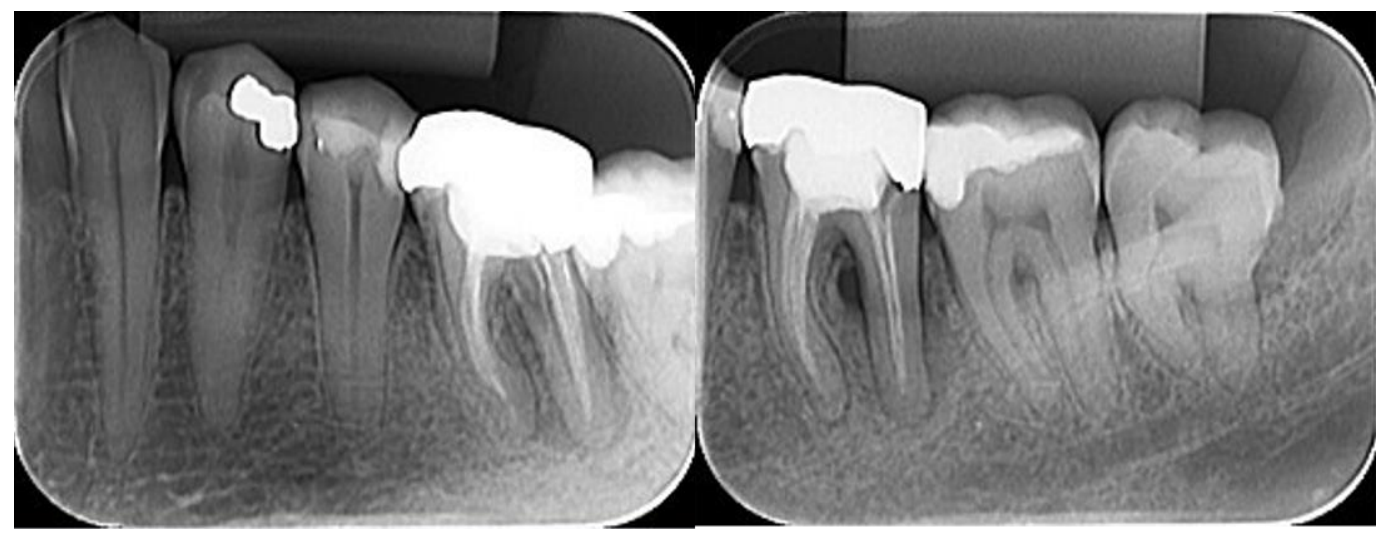

Figura 1 - Radiografia periapical com presença de rarefação óssea periapical, em periodonto lateral e região de furca no dente 36 .

Optou-se pela realização de uma TCFC de alta resolução do dente 36 no tomógrafo PreXion® 3D(Teracom, San Mateo, CA) com tamanho de voxel de $0,05 \mathrm{~mm}$ e FOV de $5 \times 5 \mathrm{~cm}$ para avaliar a possibilidade de trinca ou fratura radicular e esta revelou dilaceração radicular no dente 36, imagem hiperdensa no interior dos condutos radiculares mésio-vestibular, mésio-lingual e distal compatível com material obturador endodôntico e linha hipodensa longitudinal, se estendendo do terço cervical ao apical, na raiz distal, compatível com fratura radicular e perda óssea associada, e lesão em furca (Figura 25).

Diante da associação do quadro clínico com os sinais dos exames por imagem, foi realizada a exodontia do dente e o planejamento para reabilitação com implante. Uma radiografia periapical foi realizada 4 meses após a exodontia e notouse sinais compatíveis com reparo ósseo (Figura 6).

\section{DISCUSSÃO}

Desde a descoberta dos raios $-x$ em 1895 por Roentgen e sua aplicação na Odontologia, o diagnóstico por imagens radiográficas tem sido amplamente utilizado na Endodontia ${ }^{8}$. A radiografia periapical é adequada para a avaliação de estruturas dentais, como coroa, raiz e estruturas adjacentes; entretanto, a literatura aponta limitações no diagnóstico de fraturas radiculares e reabsorções internas e externas $^{9}$. No presente caso, a radiografia periapical foi realizada e permitiu avaliar presença de alterações no dente 36, no entanto, o diagnóstico da fratura radicular não foi possível nessa técnica radiográfica. 


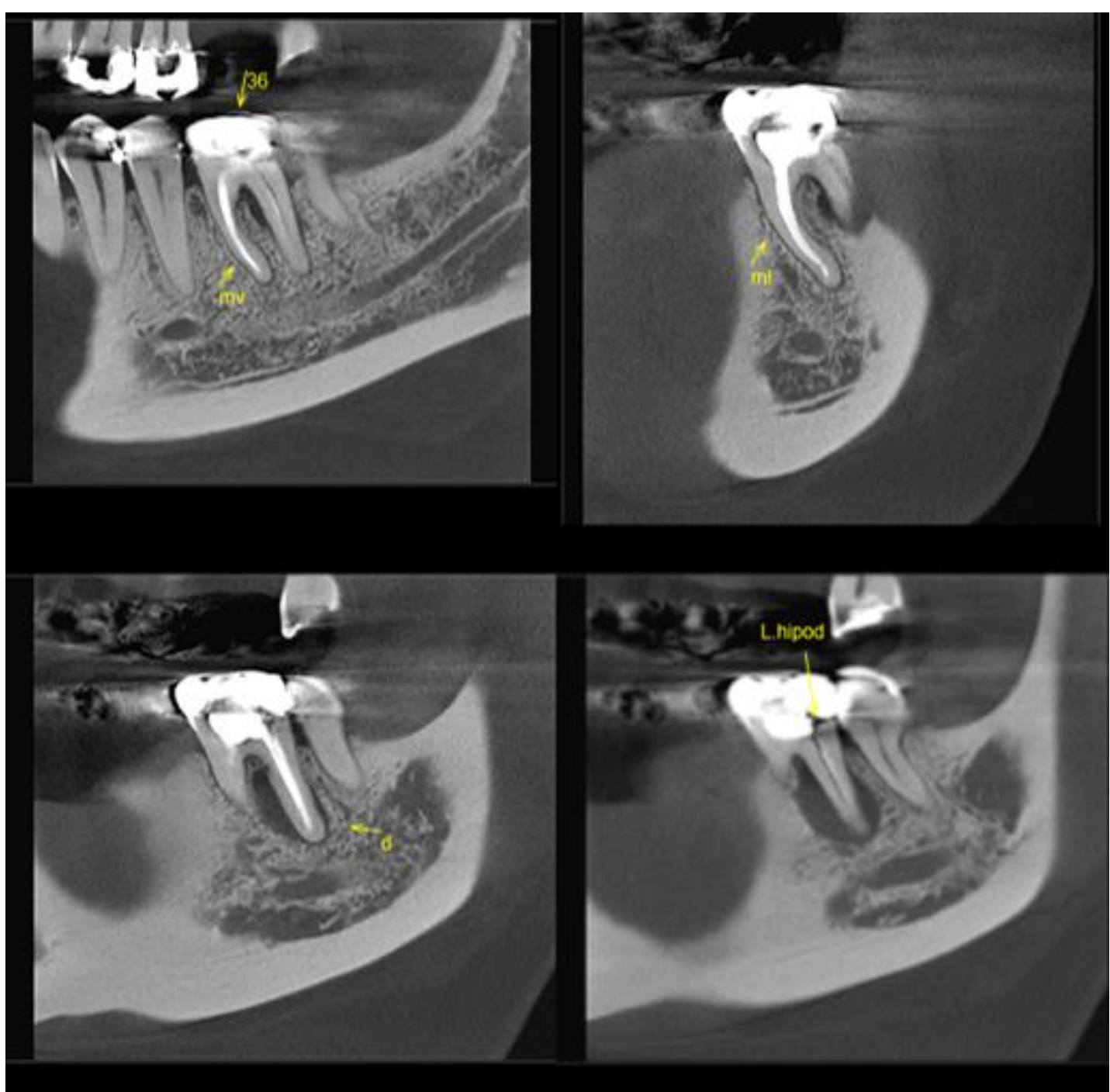

Figura 2 - Tomografia computadorizada feixe cônico: cortes vestíbulo-linguais evidenciando perda óssea em região de periápice do dente 36 .

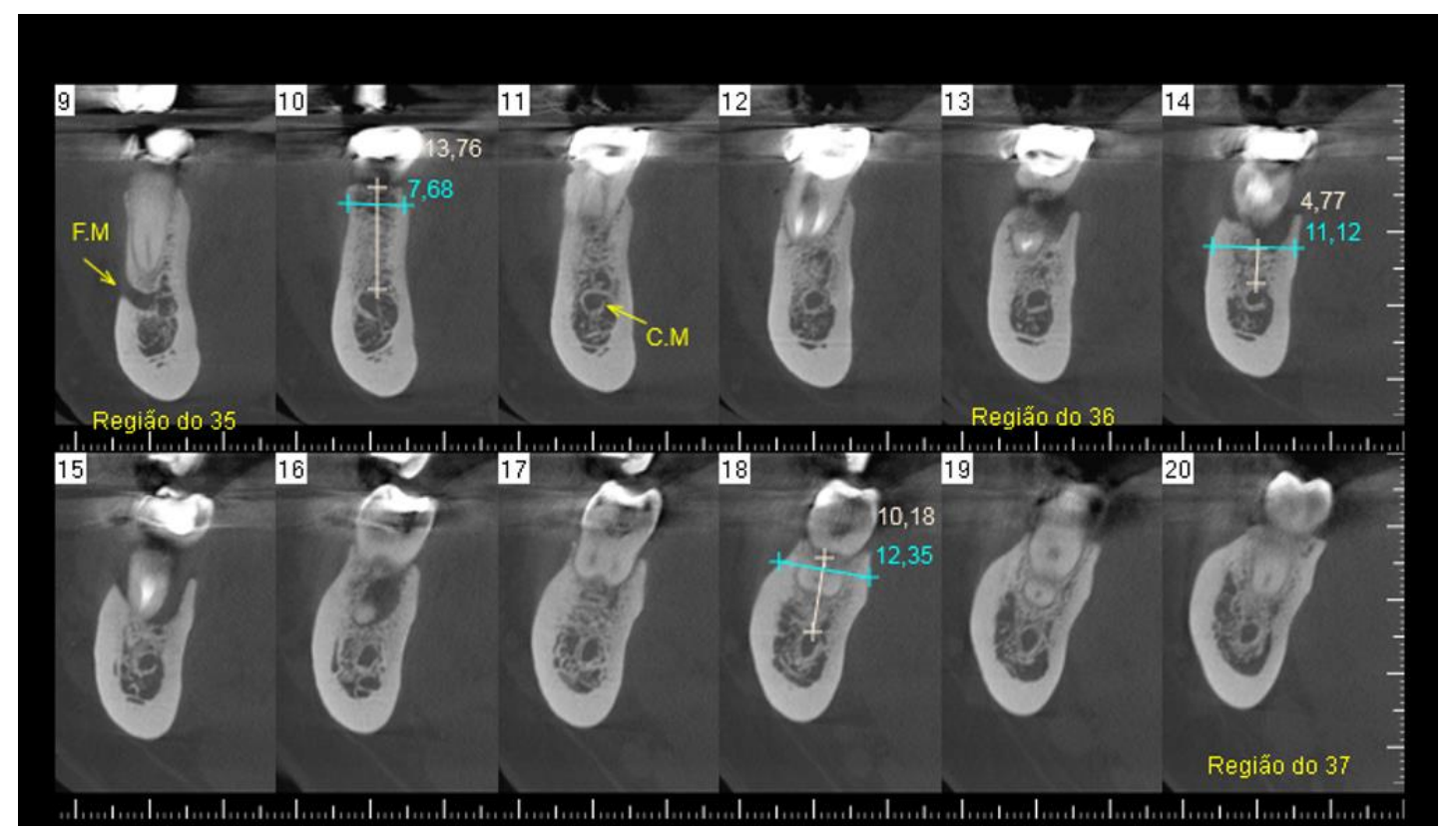

Figura 3 - Cortes sagitais de TCFC evidenciando área hipodensa em periodonto e furca (36) e presença de linha hipodensa do terço cervical ao apical, na raiz distal, compatível com fratura. 


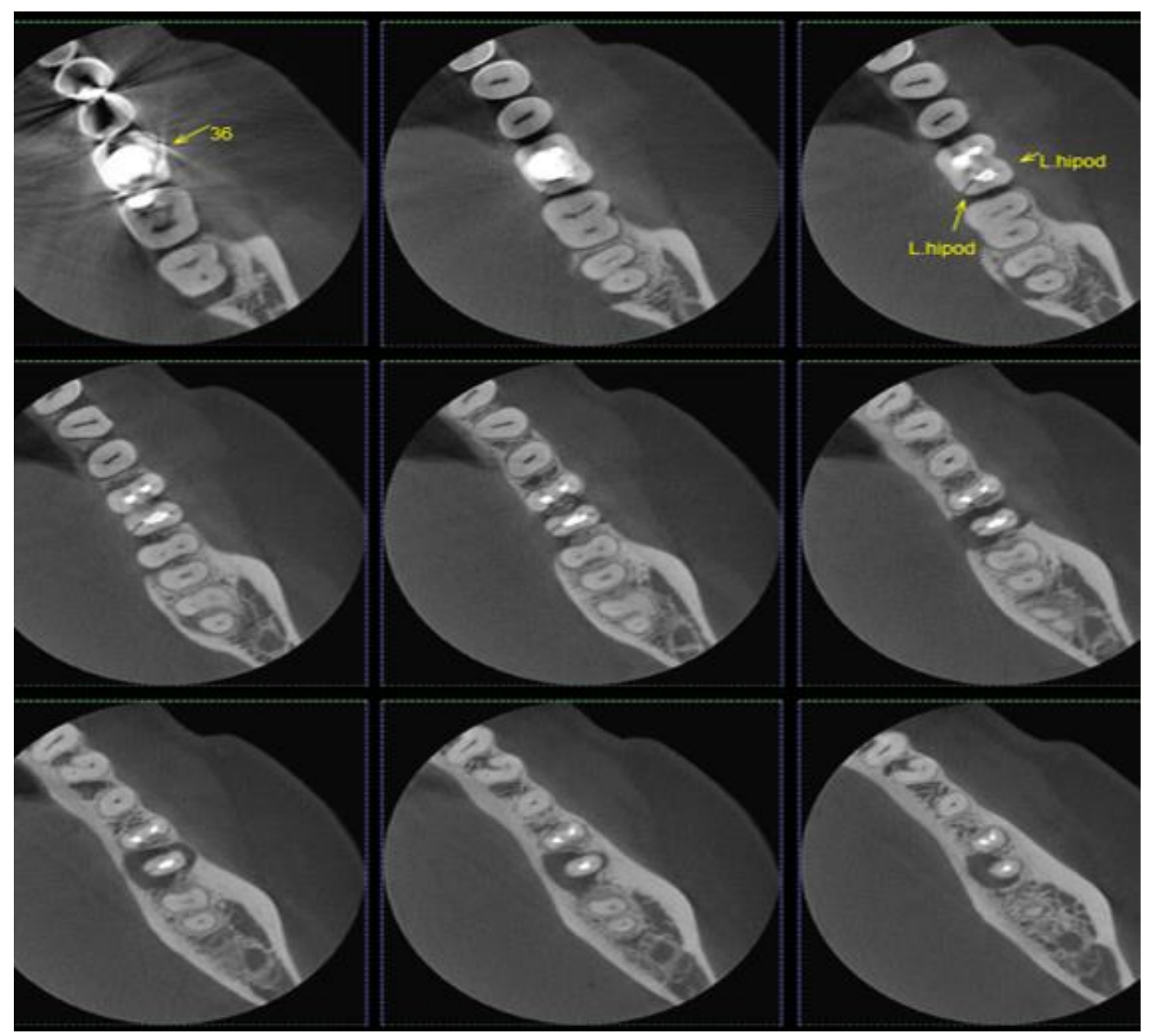

Figura 4 - Sequência de cortes axiais e TCFC evidenciando presença de linha hipodensa do terço cervical ao apical, na raiz distal, compatível com fratura e perda óssea, associada com lesão em furca (36).

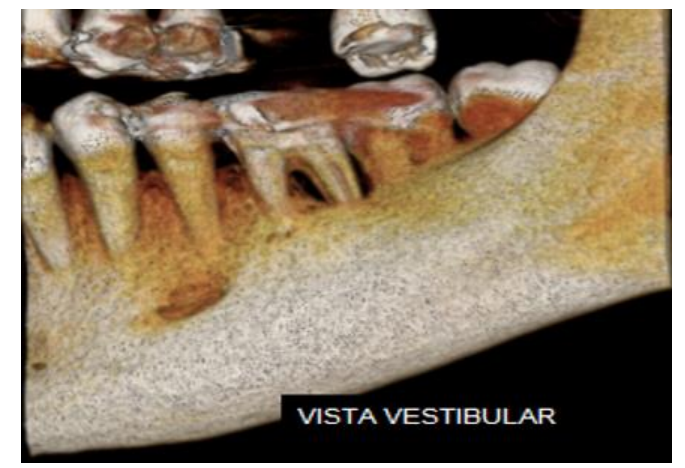

Figura 5 - Reconstrução tridimensional em vista vestibular do lado esquerdo representativa de perda óssea alveolar no dente 36 .

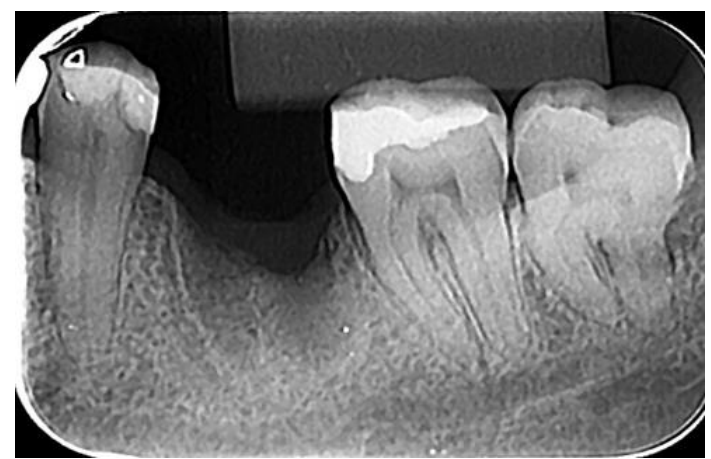

Figura 6 - Radiografia periapical pós exodontia do dente 36 evidenciando neoformacão óssea em alvéolo. 
As fraturas radiculares são fraturas envolvendo somente as raízes dentárias, mas que podem se estender desde a porção apical até a parte coronária do dente envolvido. Podem acontecer em diferentes angulações e planos, e podem dividir os segmentos dentários em duas porções quando há a separação total dos fragmentos ${ }^{10}$. No caso em questão, foi possível diagnosticar uma fratura longitudinal se estendendo desde o terço cervical ao apical, na raiz distal do dente 36 , no entanto, não atingiu a porção coronária, não causando o deslocamento de fragmento.

Vários fatores têm sido atribuídos como causa das fraturas, como dentes tratados endodonticamente com restaurações extensas, presença de retentores intrarradiculares e dentes com rizogênese incompleta que sofreram traumatismos. Com isso tem se tentado estabelecer um protocolo para minimizar esses acidentes, como acessos coronários mais conservadores, uso de retentores que não necessitem grandes desgastes da estrutura dentária e sempre que indicado, tentar promover a apicigênese para a correta formação do ápice radicular no lugar do tratamento endodôntico convencional ${ }^{11}$. Diferentemente dos acessos coronários conservadores, neste caso foi possível notar uma grande destruição coronária no dente 36 , o que não contribuiu com manutenção de estrutura dentária.

A tomografia computadorizada de feixe cônico tem sido frequentemente utilizada no diagnóstico de fraturas radiculares, lesões periapicais, cirurgias parendodônticas e planejamentos para utilização de implantes. A resolução das imagens em três dimensões fornecidas pela TCFC também possibilita a análise da articulação temporomandibular e reabsorções, tanto internas quanto externas $^{12}$. No caso apresentado, a TCFC foi utilizada como complemento aos aspectos clínicos no diagnóstico da fratura radicular. Como vantagens da TCFC em comparação a radiografia periapical convencional está a visualização de múltiplas imagens da região de interesse em três diferentes planos do espaço e a ausência de sobreposição de estruturas por se tratar de imagem seccional, o que favorece a visualização da estrutura radicular não observada durante o exame físico. Além disso, é possível avaliar as relações entre as raízes dentárias e estruturas anatômicas adjacentes como 0 seio maxilar e o canal da mandíbula ${ }^{13,14}$.

Apesar das vantagens da TCFC, existem algumas limitações, como a presença de artefatos gerados em decorrência de materiais de alta densidade na região de interesse do exame ${ }^{3,7}$, como os materiais obturadores endodônticos no interior do canal radicular $^{2}$. Alguns tomógrafos apresentam filtros para redução desses artefatos de imagem, como 0 equipamento utilizado neste caso PreXion ${ }^{\circledR}$ 3D (Teracom, San Mateo, CA). Outra limitação associada à TCFC é a falta de treinamento do profissional que solicitou 0 exame em interpretar os achados na imagem e ainda a falta de acesso a este recurso seja pela ausência de disponibilidade deste em alguns municípios ou até mesmo no acesso impossibilitado do paciente frente ao custo do exame ${ }^{15}$.

Segundo Venkuskonis et al, 20147, fraturas radiculares horizontais são mais 
fáceis de se detectar comparadas as fraturas longitudinais, em especial as presente no plano mesiodistal. $O$ caso apresentado constatou se tratar de uma fratura longitudinal, o que dificultou seu diagnóstico na radiografia periapical. Quanto maior a resolução do aparelho de TCFC incluindo o menor tamanho de voxel, maior a sua acurácia na detecção de fraturas ${ }^{5}$, o que implica que uma imagem de baixa resolução, gerada por alguns equipamentos de tomografia, não apresentam acurácia maior comparada a uma radiografia convencional. O desenvolvimento de tomógrafos mais avançados e softwares desenvolvidos para reduzir a quantidade de artefatos causados pela presença de retentores intrarradiculares e materiais obturadores, favorecem o diagnóstico e prognóstico das fraturas radiculares $^{6}$, assim como observado no relato.

De acordo com a Associação Americana de Endodontia e a Academia Americana de Radiologia Oral e Maxilofacial ${ }^{16}$, todas as imagens radiográficas devem ser justificadas e avaliadas quanto aos benefícios e riscos para cada paciente envolvido, devendo ser considerada nesta análise a dose de radiação envolvida em cada caso. Considerando as tomografias computadorizadas de feixe cônico, as mesmas não devem ser solicitadas antes de um correto exame clinico e avaliação da história pregressa do paciente. A tomografia deve ser considerada um método complementar de diagnóstico por imagem nos casos em que a radiografia convencional não fornecer as informações necessárias ao diagnóstico, conforme observado no presente caso.

Faz-se importante salientar que a TCFC não pode ser considerada um substituto obrigatório para as radiografias periapicais e que essas apresentam um valor no diagnóstico endodôntico, além de apresentarem menor dose de radiação ao paciente e um baixo custo ${ }^{6,7}$. Entretanto, a TCFC é um recurso viável para diagnóstico endodôntico que pode ser especialmente empregada em casos de suspeita de fraturas radiculares.

\section{CONSIDERAÇÕES FINAIS}

Pode-se concluir que a tomografia computadorizada por feixe cônico contribuiu para o diagnóstico da fratura radicular, e estabelecimento do correto plano de tratamento para o caso apresentado, sendo a mesma apontada pela literatura como uma importante ferramenta para o cirurgiãodentista, em especial na prática da Endodontia.

\section{ABSTRACT}

Aim: Root fractures are a type of trauma that can compromise the dental element, and if not properly diagnosed and treated, can lead to the loss of the fractured teeth. This paper aims to present a case of application of CBCT in the diagnosis of root fracture. Case Report: Patient 50 years old, female, attended the dental office with complaint of painful symptomatology on tooth 36 , and had been perceived for 3 months, with mild worsening during the reported period. It was reported a history of endodontic treatment in tooth 36 , 1 year ago. Intraoral examination revealed pain to percussion. After a clinical examination, a periapical radiograph revealed periapical bone rarefaction in the lateral periodontium at the cervical region of the tooth 36, vertical loss of the alveolar bone crest on the distal face. A CBCT of the tooth 36 was performed to evaluate the possibility of cracking or root fracture and this revealed a hyperdense image inside the mesiobuccal, mesio-lingual and distal root conduits compatible with endodontic filling material and longitudinal hypodense line, extending from the cervical to the apical third, at the distal root, compatible with fracture and associated bone loss, with furcation. Considering the diagnosis, tooth extraction and planning 
for implant rehabilitation were performed. A periapical radiography was performed 4 months after the extraction and signs compatible with bone repair were noted. Final Considerations: CBCT contributed to the diagnosis of root fracture, and established the correct treatment plan for the case.

\section{KEYWORDS}

Endodontics; Diagnosis;Tomography, X-Ray Computed.

\section{REFERÊNCIAS}

1. Guangning Z, Jiyao L. Radiographic diagnosis of vertical root fracture. Hua $\mathrm{Xi}$ Kou Qiang Yi Xue Za Zhi. 2016 Feb;34(1):15 .

2. Wanderley VA, Neves FS, Nascimento MCC, Monteiro GQM, Lobo NS, Oliveira ML, Nascimento Neto JBS, Araujo LF. Detection of Incomplete Root Fractures in Endodontically Treated Teeth Using Different High-resolution Cone-beam Computed Tomographic Imaging Protocols. J Endod. 2017 Oct;43(10):1720-1724.

3. Neves FS, Freitas DQ, Campos PS, Ekestubbe A, Lofthag-Hansen S. Evaluation of cone-beam computed tomography in the diagnosis of vertical root fractures: the influence of imaging modes and root canal materials. J Endod. 2014 Oct;40(10):15306.

4. Hassan B, Metska ME, Ozok AR, van der Stelt $\mathrm{P}$, Wesselink PR. Comparison of five cone beam computed tomography systems for the detection of vertical root fractures. J Endod. 2010 Jan;36(1):126-9.

5. Elsaltani $\mathrm{MH}$, Farid MM, Eldin Ashmawy MS. Detection of Simulated Vertical Root Fractures: Which Cone-beam Computed Tomographic System Is the Most Accurate?J Endod. 2016 Jun;42(6):972-7.

6. Silveira PF, Vizzotto MB, Liedke GS, da Silveira HL, Montagner F, da Silveira HE. Detection of vertical root fractures by conventional radiographic examination and cone beam computed tomography - an in vitro analysis. Dent Traumatol. 2013 Feb;29(1):41-6.

7. Venskutonis T, Plotino G, Juodzbalys G, Mickevičienè $L$. The importance of conebeam computed tomography in the management of endodontic problems: a review of the literature. J Endod. 2014 Dec;40(12):1895-901.

8. Aminoshariae A, Kulild JC, Syed A. Conebeam Computed Tomography Compared with Intraoral Radiographic Lesions in Endodontic Outcome Studies: A Systematic Review. J Endod. 2018 Nov;44(11):16261631.

9. Tamse A, Kaffe I, Lustig J, Ganor Y, Fuss Z. Radiographic features of vertically fractured endodontically treated mesial roots of mandibular molars. Oral Surg Oral Med Oral Pathol Oral Radiol Endod. 2006 Jun;101(6):797-802.

10. Baageel TM, Allah EH, Bakalka GT, Jadu F, Yamany I, Jan AM, Bogari DF, Alhazzazi TY. Vertical root fracture: Biological effects and accuracy of diagnostic imaging methods. J Int Soc Prev Community Dent. 2016 Aug;6(Suppl 2):S93-S104.

11. Makeeva IM, Byakova SF, Novozhilova NE. Risk factors for vertical root fractures after endodontic treatment. Stomatologiia (Mosk). 2016;95(6):67-70.

12. Bernardes RA, de Moraes IG, Húngaro Duarte MA, Azevedo BC, de Azevedo JR, Bramante CM. Use of cone-beam volumetric tomography in the diagnosis of root fractures. Oral Surg Oral Med Oral Pathol Oral Radiol Endod. 2009 Aug;108(2):270-7.

13. Corbella S, Del Fabbro M, Tamse A, Rosen E, Tsesis I, Taschieri S. Cone beam computed tomography for the diagnosis of vertical root fractures: a systematic review of the literature and meta-analysis. Oral Surg Oral Med Oral Pathol Oral Radiol. 2014 Nov;118(5):593-602.

14. Metska ME, Aartman IH, Wesselink PR, Özok AR. Detection of vertical root fractures in vivo in endodontically treated teeth by cone-beam computed tomography scans. J Endod. 2012 Oct;38(10):1344-7.

15. Cotton TP, Geisler TM, Holden DT, Schwartz SA, Schindler WG. Endodontic applications of cone-beam volumetric tomography. J Endod 2007;33:1121-32.

16. American Association of Endodontists; American Academy of Oral and Maxillofacial Radiology. Use of cone-beam computed tomography in endodontics Joint Position Statement of the American Association of Endodontists and the American Academy of Oral and Maxillofacial Radiology. Oral Surg Oral Med Oral Pathol Oral Radiol Endod. 2011 Feb;111(2):234-7. 